\title{
Diretrizes para o uso de Veículo Aéreo não Tripulado (VANT) Para Inspeção de Segurança em Canteiros de Obra
}

Roseneia Rodrigues Santos de Melo

Dayana Bastos Costa Juliana Sampaio Álvares

\section{Introdução}

A indústria da construção civil ainda enfrenta dificuldades quanto à gestão da segurança nos canteiros de obras, cuja ineficiência está diretamente relacionada à ocorrência de acidentes. Embora a inspeção seja um elemento importante do sistema de gestão de segurança, responsável pela detecção e correção dos riscos (WOODCOCK, 2014), ainda se observam deficiências quanto a este processo, tais como a ausência de procedimento estruturado para conduzir a inspeção (LIN et al., 2014), supervisão insuficiente e a dificuldade em visualizar as condições de trabalho (SHRESTHA et al., 2011).

Para Irizarry et al., (2012), o processo de inspeção de segurança na construção civil deve possuir três características principais: frequência, observação direta e interação direta com os funcionários. Para isso, devem ser estabelecidas rotinas de inspeção consistentes, baseando-se em critérios e requisitos de segurança estipulados por normas e regulamentações. Entretanto, o tamanho do canteiro e a quantidade de 
atividades a serem observadas influenciam no tempo gasto para a avaliação de suas condições (CAMBRAIA et al., 2010; IRIZARRY et al., 2012).

Além disso, determinados estudos (KIM et al., 2008; PARK et al., 2013) e a prática mostram que os processos de monitoramento e inspeção gerencial apresentam uma série de problemas, que acabam por reduzir a eficiência e eficácia destas avaliações. Segundo os autores citados, tais problemas estão associados a: (a) insuficiência de pessoal para análise dos requisitos de segurança e a alta demanda de preenchimento manual de dados; (b) excesso de trabalho na coleta de dados, devido ao grande número de requisitos a serem avaliados; (c) falta de padronização dos checklists de avaliação, bem como dos meios de processamento e análise dos dados; (d) perdas de informação entre coleta e processamento de dados; (e) pouca comunicação entre os intervenientes do projeto; (f) dificuldade de agir em tempo real para corrigir problemas e de realizar ações preventivas.

Em vista disso, e com o surgimento de novas tecnologias, muitos pesquisadores têm buscado conhecer o potencial delas para a melhoria dos processos gerenciais de construção. Estudos recentes aplicados a domínios da engenharia civil apontam que os Veículos Aéreos Não Tripulados (VANTs), com câmeras digitais acopladas, podem fornecer informações visuais em alta resolução, contribuindo com o monitoramento em tempo real (THEMISTOCLEOUS et al., 2014).

O VANT, conhecido, em inglês, como Unmanned Aerial Vehicles/Systems (UAV/ UAS), é definido como toda aeronave projetada para operar sem piloto a bordo (IRIZARRY et al., 2012). Inicialmente desenvolvido para fins militares, o VANT tem sido empregado em diferentes domínios da engenharia nos últimos anos. Na engenharia civil, destacam-se aplicações para inspeção de rodovias (ZHANG, 2008); inspeção de danos e rachaduras (ESCHMANN et al., 2012); monitoramento de patologias em fachada (EMELIANOV et al., 2014); e inspeção de pontes (METNI; HAMEL, 2007).

Além de tais aplicações, estudos realizados por Irizarry et al. (2012) e Irizarry e Costa (2016) têm explorado o potencial dessa tecnologia como uma ferramenta para auxiliar na gestão da segurança dentro dos canteiros de obras. Para Kim e Irizarry (2015), o VANT pode contribuir na redução do tempo gasto para realizar tarefas de monitoramento, na melhoria do desempenho geral do projeto e na agilidade do processo de reação aos potenciais riscos. Tal potencialidade está diretamente relacionada ao baixo custo, à alta mobilidade, à velocidade e à segurança oferecida pela ferramenta durante o procedimento de aquisição de dados (imagens e vídeos). Além disso, o VANT pode diminuir os custos operacionais envolvidos no processo de inspeção e ser utilizado em situações em que uma inspeção tripulada não seja possível. (MORGENTHAL; HALLERMANN, 2014).

Embora haja grandes expectativas quanto ao impacto econômico do crescimento da indústria de tecnologia VANT no Brasil e no mundo, a falta de regulamentação para 
o uso comercial da aeronave é um dos grandes entraves para a sua disseminação. No Brasil, as atividades de aviação comercial são reguladas e monitoradas pela Agência Nacional de Aviação Civil (ANAC). Segundo a proposta de regulamentação da ANAC (2015), os critérios exigidos para a operação legal do VANT variam de acordo com a classificação das aeronaves, baseada em seu Peso Máximo de Decolagem (PMD), nos tipos de operação e de acordo com os critérios visuais.

Apesar das recentes aplicações da tecnologia VANT para a gestão de obras, ainda se desconhece qual o seu impacto no processo de inspeção da segurança. Este capítulo visa avaliar a aplicabilidade do Veículo Aéreo Não Tripulado para inspeção de segurança em canteiros de obra. Para tal, foram desenvolvidos estudos de caso com a realização de voos experimentais e elaboração de protocolo para sistematizar o processo de inspeção.

\section{Método de Pesquisa}

A pesquisa foi dividida nas seguintes etapas: (a) revisão da literatura; (b) adaptação de protocolos de pesquisa e escolha da tecnologia VANT; (c) estudos de caso e (d) avaliação da aplicabilidade do VANT para inspeção de segurança e análise prévia da não conformidade dos requisitos de segurança dos elementos inspecionados.

Foram adaptados para o contexto brasileiro os protocolos para uso de VANT em monitoramento de obras, com ênfase em inspeção de segurança, desenvolvidos nos estudos de Irizarry et al. (2015), conforme a seguir:

- Formulário de Planejamento: informações gerais da obra e planejamento do voo;

- Checklist para Missão VANT: checklist de procedimentos para realização dos voos em condições de segurança e cadastro de informações operacionais;

- Checklist de Segurança segundo o tipo de captura: checklist adaptado às normas NR 18 - Condições e Meio Ambiente de Trabalho na Indústria da Construção e NR 35 - Trabalho em Altura. Inicialmente, foram selecionados os itens relativos às normas que necessitam de verificação visual e estão situados na área externa das construções. Esses itens foram divididos em categorias de acordo com o tipo de captura, quais sejam: (1) Overview - visão geral do canteiro, com ênfase em limpeza e organização de canteiro, instalações temporárias e gestão de resíduos; (2) Medium View - requisitos de segurança que envolvem Equipamentos de Proteção Coletiva (EPC) e Equipamentos de Proteção Individual (EPI); (3) Close Up View - avalia os requisitos de segurança por serviço, tais como cobertura, concretagem e alvenaria, operação de equipamentos e fachada. No total, o checklist contemplou 45 itens. 
Além disso, foi desenvolvida uma versão do Checklist de Segurança para coleta em campo, no qual se apresenta o resumo dos itens de segurança a serem avaliados (total de 24 itens), usado para orientar o piloto e observá-lo durante a coleta de dados com o VANT.

O equipamento selecionado para realização do estudo foi um DJI Phantom 3 Advanced (Figura 1), com câmera Sony EXMOR 1/2.3" acoplada, que possui resolução de 12,76 megapixels, tamanho de imagem de $4000 \times 3000$, gerando fotos nos formatos JPEG e DNG e vídeos em MP4. Tal escolha justifica-se pelo fato desse modelo de VANT ser uma tecnologia acessível e comercial, facilitando sua adoção por construtoras e empresas de consultoria. Além disso, utilizou-se um conjunto de 3 baterias visando garantir a autonomia de voo necessária.
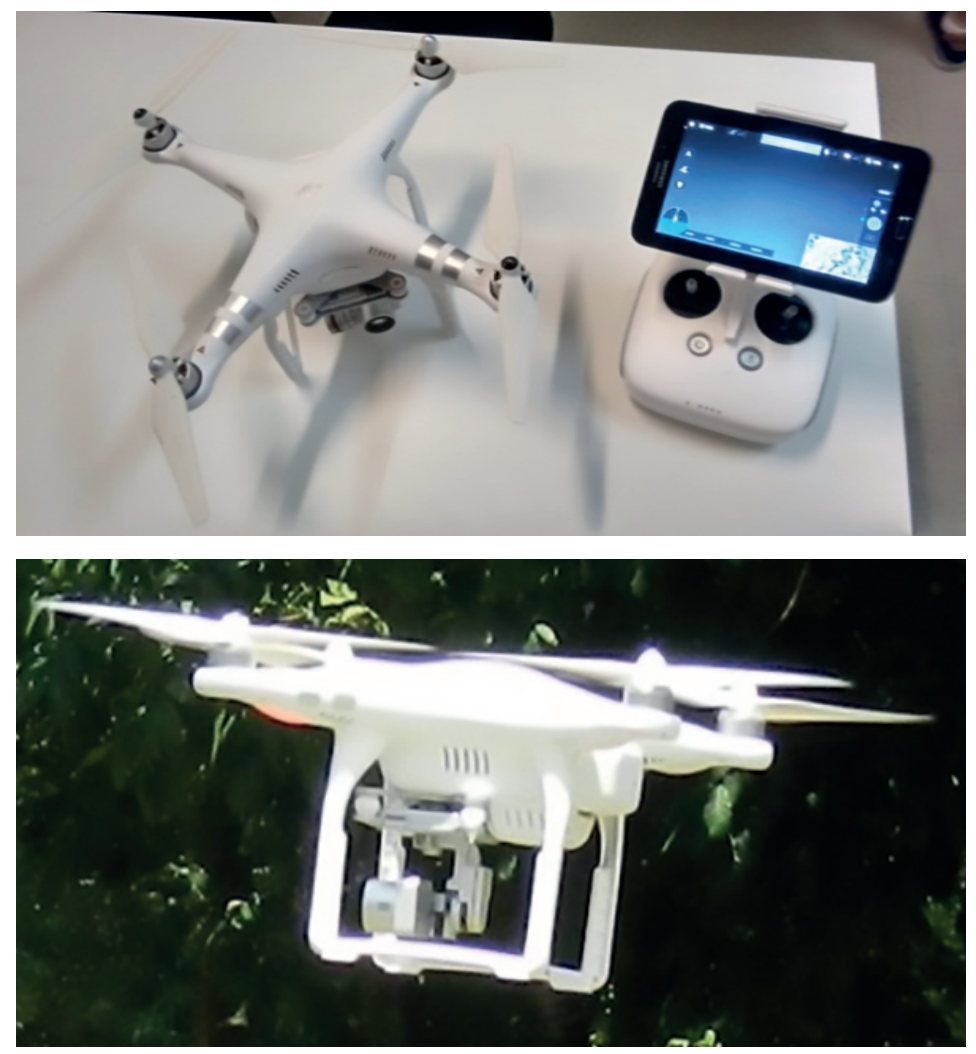

Figura 1 - DJI Phantom 3 Advanced. Fonte: Projeto de Pesquisa GETEC/UFBA.

O estudo foi realizado em duas obras residenciais, cujas características estão apresentadas na Tabela 1. 
Tabela 1 - Características dos Estudos de Caso.

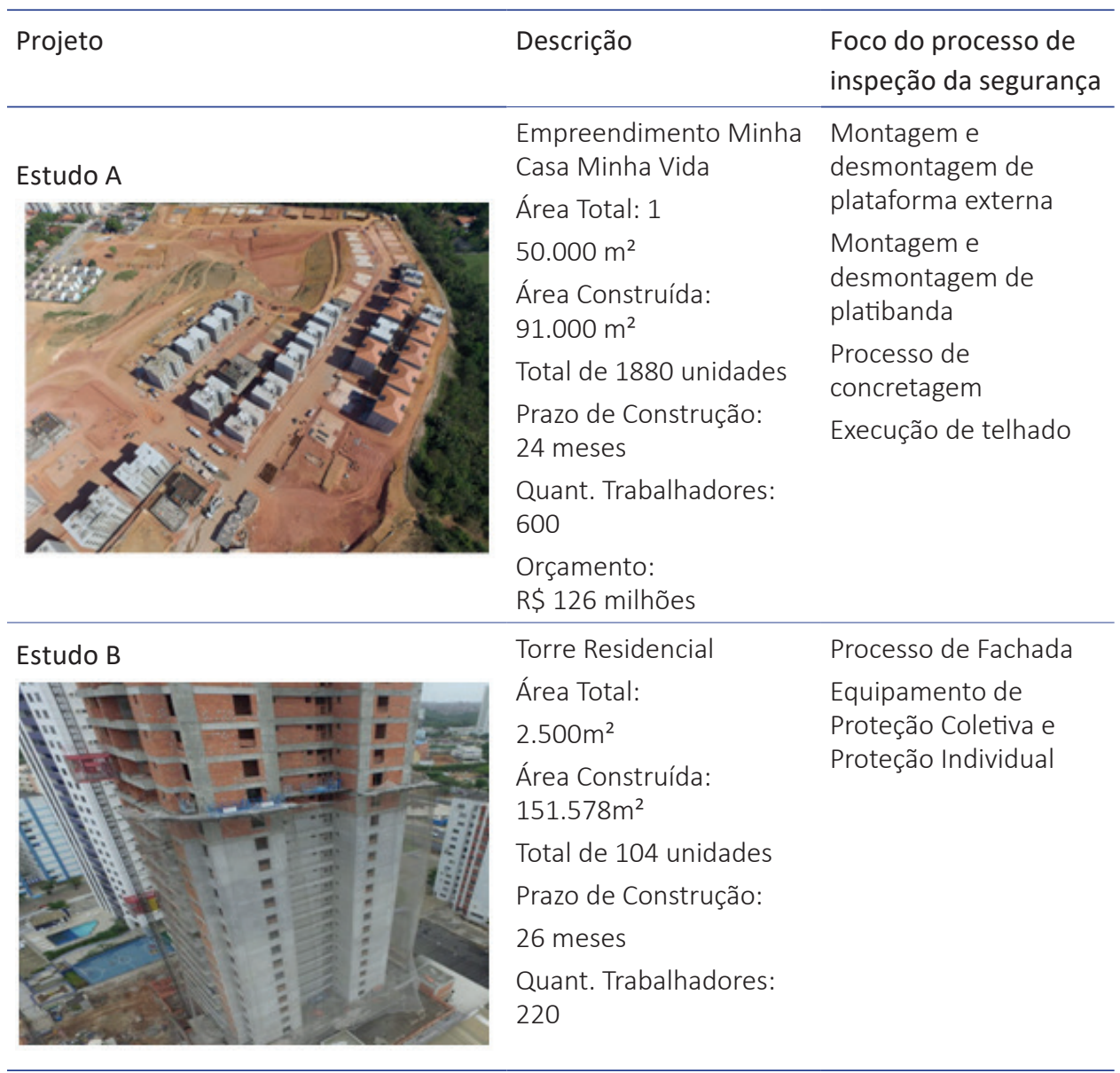

O processo de monitoramento e inspeção de segurança com o uso do VANT foi estruturado em quatro etapas: (1) planejamento; (2) coleta de dados com VANT; (3) processamento; e (4) análise de dados, conforme apresentado na Figura 2.

No total, foram realizados 26 voos com duração média de 9 minutos cada. Os dados coletados foram organizados em banco de dados. Para todos os voos, pelo menos três membros da equipe de pesquisa foram envolvidos: o piloto, o primeiro observador, que auxiliou o piloto na coleta de dados de inspeção de segurança, e o segundo observador, que se concentrou nos requisitos relativos à segurança do voo. Após os voos, em cada visita realizada, foram feitas reuniões de feedback com a equipe do projeto, para a avaliação imediata das não conformidades observadas. A Tabela 2 apresenta um resumo dos dados coletados. 


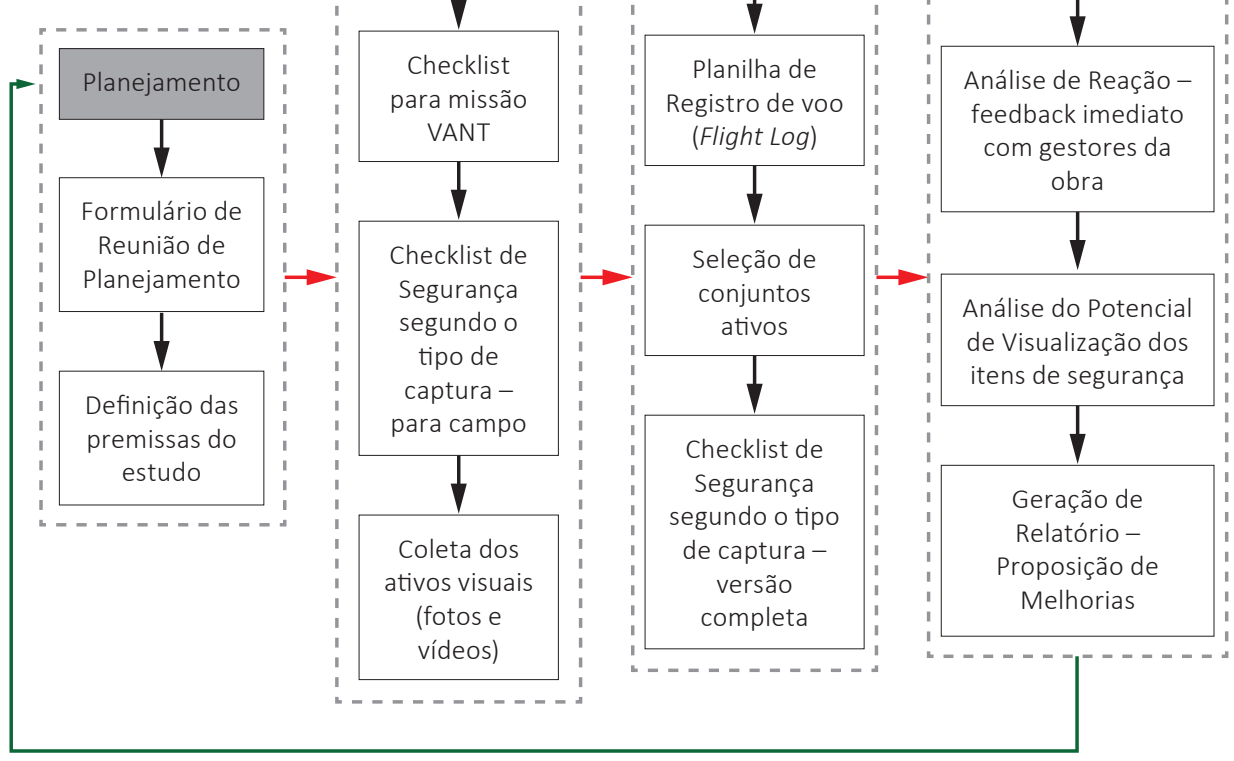

Figura 2 - Protocolo de inspeção de segurança com VANT.

Tabela 2 - Ativos visuais (fotos e vídeos) coletados durante os voos.

\begin{tabular}{llllllll}
\hline $\begin{array}{l}\text { Projeto } \\
\text { visitas }\end{array}$ & $\begin{array}{l}\text { № de } \\
\text { voos }\end{array}$ & $\begin{array}{l}\text { № de } \\
\text { fotos de }\end{array}$ & $\begin{array}{l}\text { Duração } \\
\text { dos } \\
\text { vídeos }(\mathrm{h})\end{array}$ & $\begin{array}{l}\text { Distância } \\
\text { máxima } \\
(\mathrm{m})\end{array}$ & $\begin{array}{l}\text { Altitude } \\
\text { máxima } \\
(\mathrm{m})\end{array}$ & $\begin{array}{l}\text { Tempo } \\
\text { duração dos } \\
\text { voos }(\mathrm{h})\end{array}$ \\
\hline A & $\begin{array}{l}\text { Outubro/15 a } \\
\text { Março/16 }\end{array}$ & 14 & 579 & $00: 39: 02$ & 734,0 & 120,0 & $02: 07: 43$ \\
\hline B & $\begin{array}{l}\text { Novembro/15a } \\
\text { Março/16 }\end{array}$ & 11 & 835 & $00: 14: 34$ & 173,5 & 60,0 & $01: 15: 43$ \\
\hline
\end{tabular}

Os dados foram processados com base no Checklist de Segurança por tipos de captura - versão completa (45 itens). No total, foram realizadas 8 inspeções, sendo 4 para o Projeto A e 4 para o Projeto B. Cada inspeção correspondeu a um dia de visita ao canteiro, com uma média de 3 voos por visita.

Para ambos os estudos, foram realizadas duas análises distintas. A primeira buscou avaliar a utilidade do VANT para o processo de inspeção, na qual foi verificado se era possível visualizar os itens de segurança por meio das fotos e vídeos. Com base nos itens 
vistos, a segunda análise consistiu na verificação da conformidade dos itens de segurança, baseando-se nos requisitos apontados pelas NR 18 - Condições e meio ambiente de trabalho na indústria da construção (BRASIL, 2015) e NR 35 - Trabalho em altura (BRASIL, 2014).

\section{Resultados e Discussão}

Esta seção apresenta os resultados relativos à aplicabilidade do VANT para inspeção de segurança e à análise da não conformidade dos requisitos de segurança.

\subsection{Aplicabilidade}

Dos 45 itens que compõem o Checklist de Segurança, em média, foram avaliados 34 itens para a Obra A e 36 itens para a Obra B. Os demais itens que compõem o Checklist não foram avaliados, pois não se aplicavam ao contexto da obra. De acordo com os dados obtidos por meio da análise dos ativos visuais coletados em canteiro com - VANT, $87 \%$ e $60 \%$ dos itens de segurança avaliados foram visualizados nos Estudos $A$ e $B$, respectivamente, conforme apresenta a Figura 3.

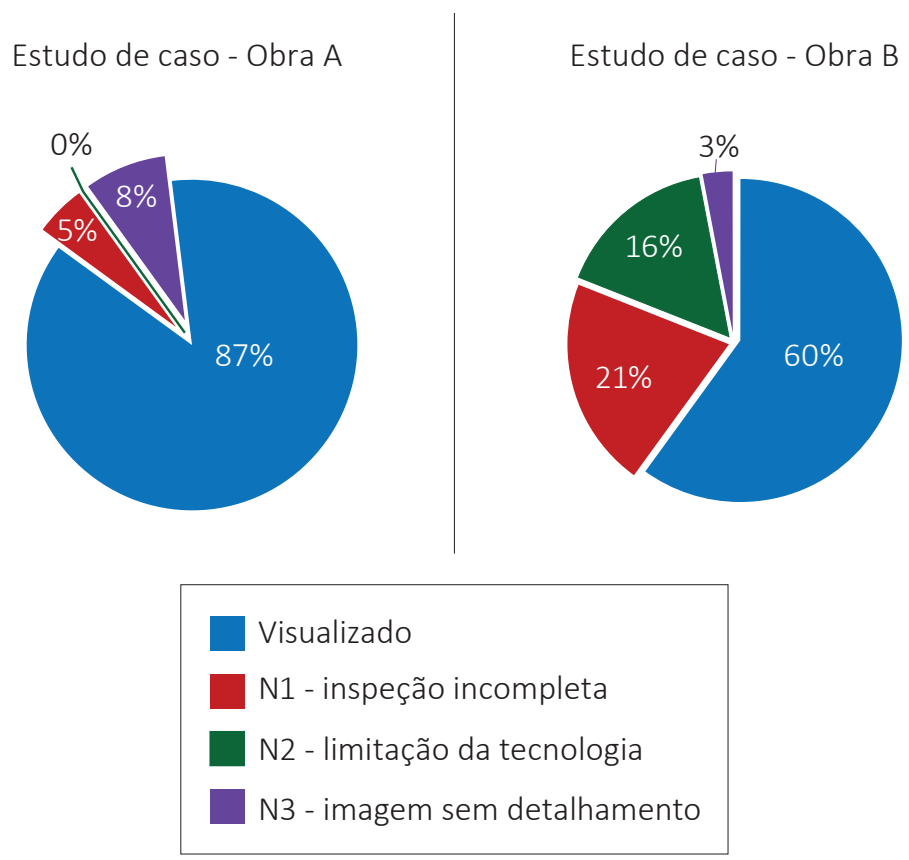

Figura 3 - Análise do Potencial de Visualização baseado no Checklist de Segurança.

No Estudo A, 5\% dos itens de inspeção de segurança não foram visualizados por motivos diretamente associados a falhas no procedimento de inspeção. Alguns 
destes itens, por alguma razão, não foram registrados nos ativos coletados em canteiro (N1 - inspeção incompleta). Destacando-se itens relacionados à montagem e desmontagem das formas, ao içamento de cargas e à sinalização e isolamento da área de movimentação de cargas. Essas falhas ocorreram devido à extensão do canteiro $\left(150.000 \mathrm{~m}^{2}\right)$ e à grande quantidade de tarefas desenvolvidas simultaneamente. Além disso, os outros $8 \%$ dos itens não visualizados ocorreram por conta de ativos coletados que não forneciam informações suficientes para a inspeção, incluindo itens como as rampas, escadas e passarelas protegidas com guarda-corpo (N3 - imagem sem detalhamento suficiente). Esses dois resultados indicam a necessidade de maior precisão na inspeção com o VANT, no que tange a um treinamento mais eficaz para piloto e observadores.

No Estudo B, devido ao caráter vertical do edifício e por possuir um canteiro restrito em termos de área livre, $16 \%$ dos itens do Checklist de Segurança não foram visualizados devido à limitação da tecnologia, tais como rampas, escadas e passarelas protegidas com guarda-corpo e itens relativos a serviços na cobertura. Além disso, $21 \%$ dos itens não visualizados foram provenientes de falhas no processo de inspeção. Entre eles, destacam-se principalmente trabalhadores protegidos de queda (guardacorpo e linha de vida), trabalhadores usando EPI, balancim, e remoção de resíduos por calhas. Em relação às barreiras que dificultaram o processo de inspeção na Obra B, ressalta-se a existência de telas de proteção ao longo da fachada, a altura limitada de voo de até $60 \mathrm{~m}$ para a área urbana, imposta por regulamentação de operação do VANT, impossibilitando a inspeção do topo do edifício de altura de $80 \mathrm{~m}$, o canteiro restrito, bem como ventos fortes no local, que limitaram a utilização do VANT por razões de segurança.

A Figura 4 apresenta os resultados da aplicabilidade segundo o tipo de captura.

Segundo a Figura 4, para a captura em Overview, foram visualizados 95\% (Estudo A) e $88 \%$ (Estudo B) dos itens do Checklist de Segurança, relativos à organização e limpeza, instalações provisórias e gestão de resíduos. Tais resultados indicam a possibilidade de visualização das condições de trabalho sob uma perspectiva global, capaz de fornecer informações para a tomada de decisão. Para a captura em Medium View, que tem como objetivo principal visualizar as proteções coletivas, houve dificuldade na aquisição de dados da Obra B, devido à existência de barreiras físicas e a condições climáticas desfavoráveis, impactando no percentual de visualização.

As capturas em Close Up, assim como no Medium View, em alguns casos tiveram seus resultados influenciados por barreiras físicas, limitação de altitude de voo, inexperiência do piloto e observadores, entre outros. Dessa forma, o Estudo B apresentou potencial de visualização de apenas 56\% em Close Up View, em função da presença de tais fatores citados. Contudo, no Estudo A, 81\% dos itens foram visualizados para o mesmo tipo de captura, por conta da menor interferência observada na aquisição de dados com o VANT. Apesar da identificação de algumas 
barreiras, os resultados foram eficazes na identificação de atos e condições inseguras para os serviços inspecionados, fornecendo feedback quase imediato para gestores e responsáveis pelas condições gerais de segurança do canteiro.

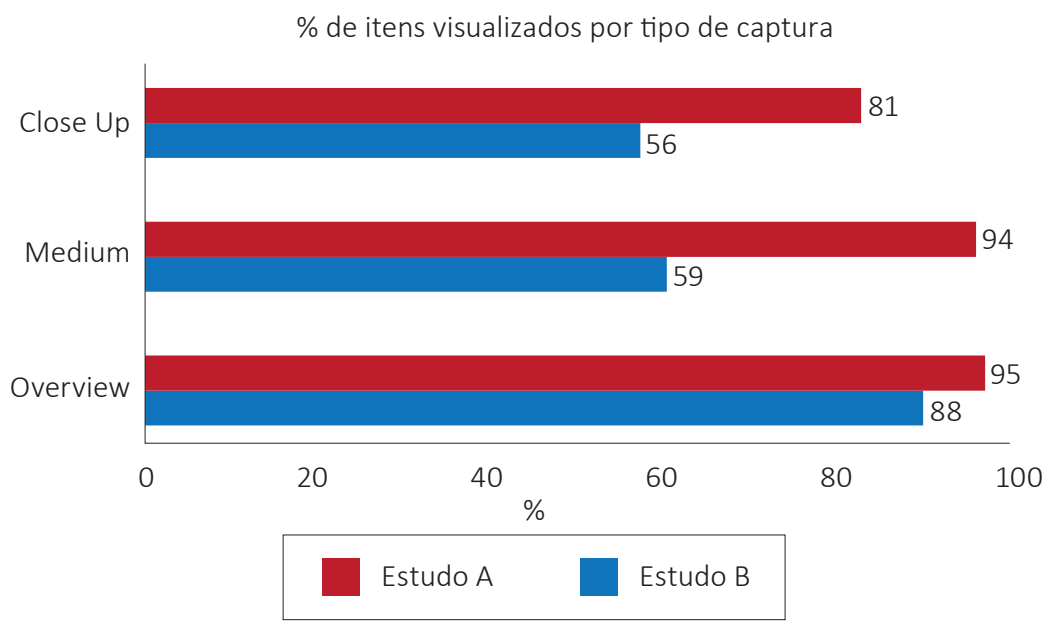

Figura 4 - Análise da aplicabilidade por tipo de captura.

\subsection{Análises das não Conformidades das Condições de Segurança}

Esta seção apresenta a análise da não conformidade dos itens de segurança inspecionados por meio dos ativos coletados com VANT nos Estudos A e B. Além disso, os resultados apresentam o status das condições de segurança no canteiro ao longo das inspeções. Para a análise das não conformidades, foram considerados apenas os itens visualizados (Figura 3), sendo 29 itens no Estudo A (que corresponde a 87\% dos 34 itens avaliados) e 21 itens no Estudo B (que corresponde a 60\% dos 36 itens avaliados). A Tabela 3 mostra um panorama do controle das não conformidades observadas nas inspeções, organizadas segundo o tipo de captura.

Tabela 3 - Análise geral do percentual de não conformidades.

\begin{tabular}{lllllllll}
\hline \multirow{2}{*}{$\begin{array}{l}\text { \% de não } \\
\text { conformidade }\end{array}$} & \multicolumn{2}{l}{ 1å Inspeção } & \multicolumn{2}{l}{ 2a Inspeção } & \multicolumn{2}{l}{ 3a Inspeção } & \multicolumn{2}{c}{ 4a Inspeção } \\
\cline { 2 - 9 } & Obra A & Obra B & Obra A & Obra B & Obra A & Obra B & Obra A & Obra B \\
\cline { 2 - 10 } & $17 \%$ & $45 \%$ & $14 \%$ & $50 \%$ & $12 \%$ & $52 \%$ & $19 \%$ & $68 \%$ \\
\hline
\end{tabular}


Observa-se que o grau de não conformidade dos itens de segurança avaliados na Obra B é expressivamente superior aos da Obra A, de modo que se tornam preocupantes as condições de segurança as quais os trabalhadores da Obra B são submetidos. Além das diferenças quanto às características físicas e construtivas, as obras diferem significativamente quanto ao sistema de gestão da segurança adotado. A Obra A possui uma equipe formada por engenheiro de segurança, 3 técnicos e 5 estagiários de segurança, com alguns procedimentos de inspeção já padronizados, como permissões de serviço diário para trabalho em altura e inspeção mensal para proteções coletivas. A obra B, no entanto, possui apenas um técnico para o desenvolvimento de todas as atividades relativas à gestão da segurança, sem nenhuma padronização dos processos. Em vista disso, tais resultados apresentados na Tabela 3 evidenciam o impacto da estruturação da gestão da segurança e seus processos nas condições de trabalho no canteiro de obra. As Tabelas 4 e 5 apresentam alguns itens avaliados na análise de não conformidade, realizada para ambos os estudos.

Tabela 4 - Itens avaliados no Checklist de Segurança (Estudo A).

\begin{tabular}{|c|c|c|c|c|c|}
\hline Item & Critério de Segurança & 1ㅁ Visita & 2a Visita & 3a Visita & 4a Visita \\
\hline \multicolumn{6}{|c|}{ 1) Tipo de Captura \# 1. Visualização geral (Overview) } \\
\hline 1.5 & $\begin{array}{l}\text { As rotas internas deverão estar limpas e } \\
\text { desobstruídas }\end{array}$ & & & & \\
\hline 1.11 & Resíduos protegidos das intempéries & $x$ & $x$ & X & X \\
\hline \multicolumn{6}{|c|}{ 2) Tipo de Captura\# 2.Visualização de Altitudes Intermediárias (Medium altitude view) } \\
\hline 2.1 & $\begin{array}{l}\text { Plataforma de proteção (bandejas) } \\
\text { instalada em toda a periferia isenta de } \\
\text { sobrecarga não prevista e com forração } \\
\text { do piso completa }\end{array}$ & $x$ & & $x$ & $x$ \\
\hline 2.2 & $\begin{array}{l}\text { Escadas de uso coletivo, rampas e } \\
\text { passarelas são dotadas de corrimão e } \\
\text { rodapé }\end{array}$ & & & & \\
\hline
\end{tabular}

3) Tipo de Captura\# 3. Visualização aproximada por tipo de serviço (Close up view)

3.1) Cobertura

3.1.1 Os trabalhadores que se encontram na cobertura estão usando EPI

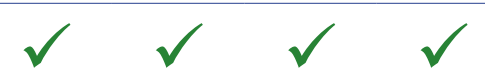

3.1.2 Os trabalhadores que se encontram na cobertura estão protegidos contra queda (cinto de segurança tipo paraquedista ligado a cabo-guia)

Legenda: $\quad \sqrt{\begin{array}{l}\text { Item } \\ \text { conforme }\end{array}} \times \begin{aligned} & \text { Item não } \\ & \text { conforme }\end{aligned}-\quad \begin{aligned} & \text { Não } \\ & \text { visualizado }\end{aligned}$




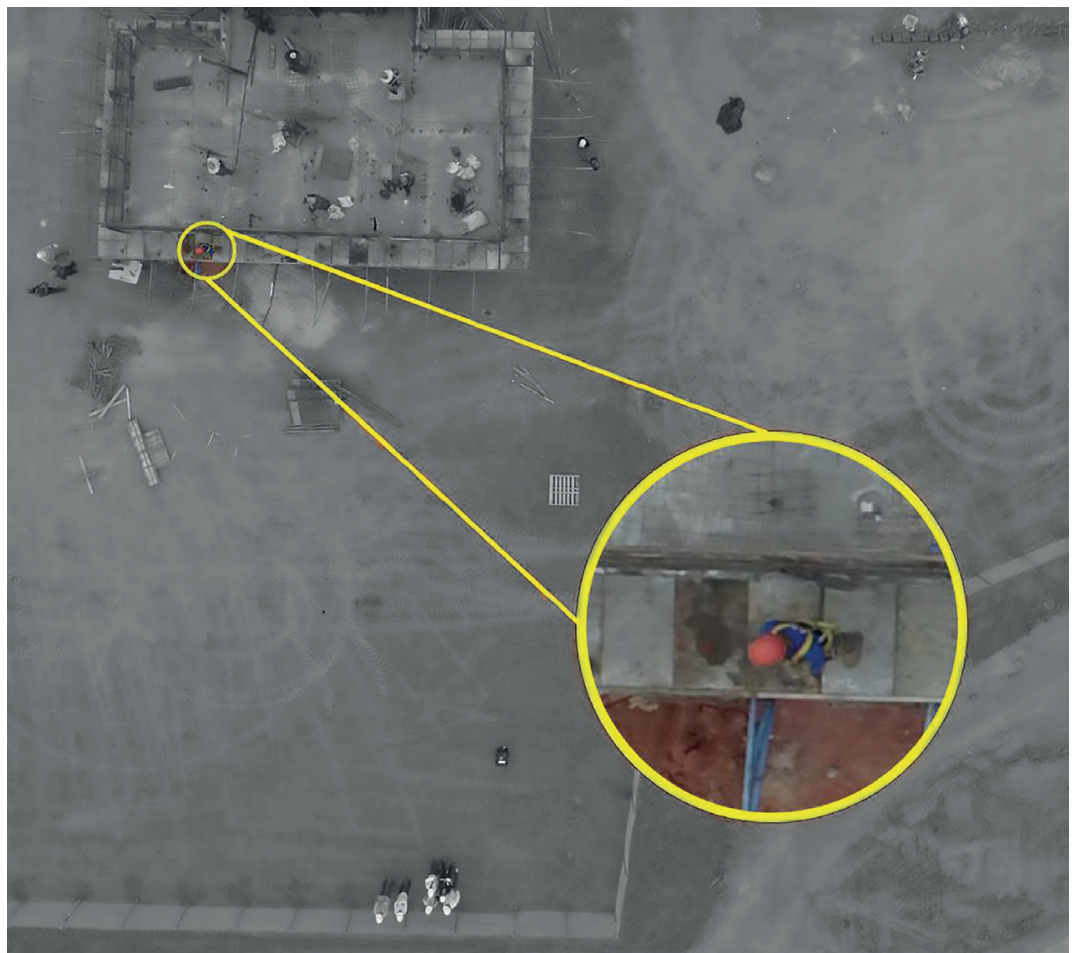

Figura 6 - Trabalhador desprotegido contra queda (sem cinto trava-quedas fixado na estrutura).

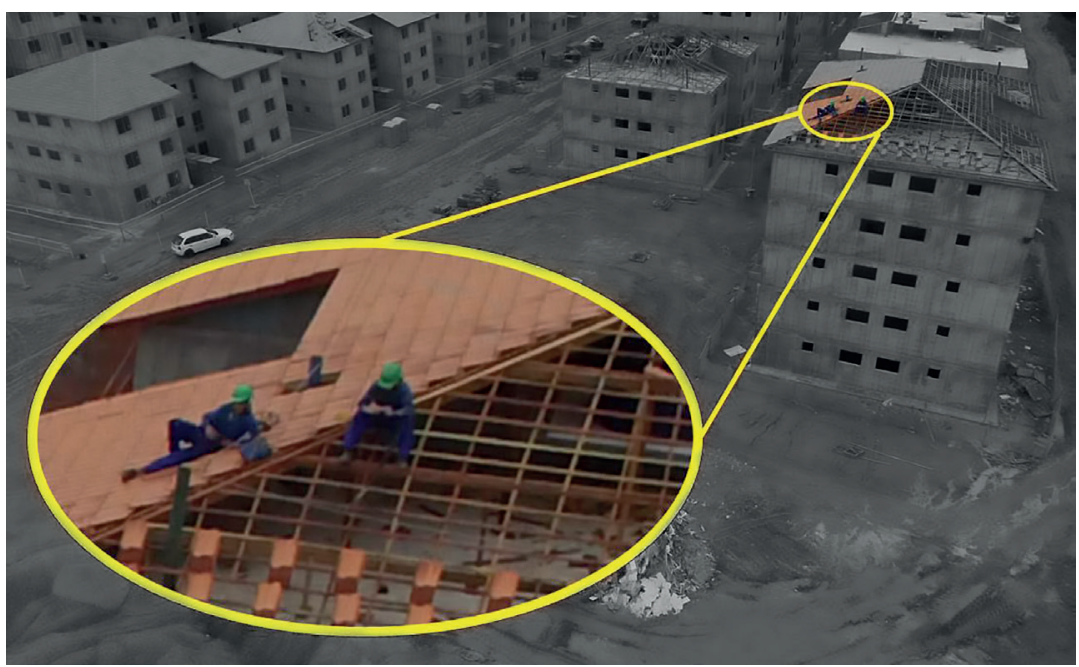

Figura 7 - Trabalhadores na cobertura desprotegidos contra queda (cinto não fixado a linha de vida). 


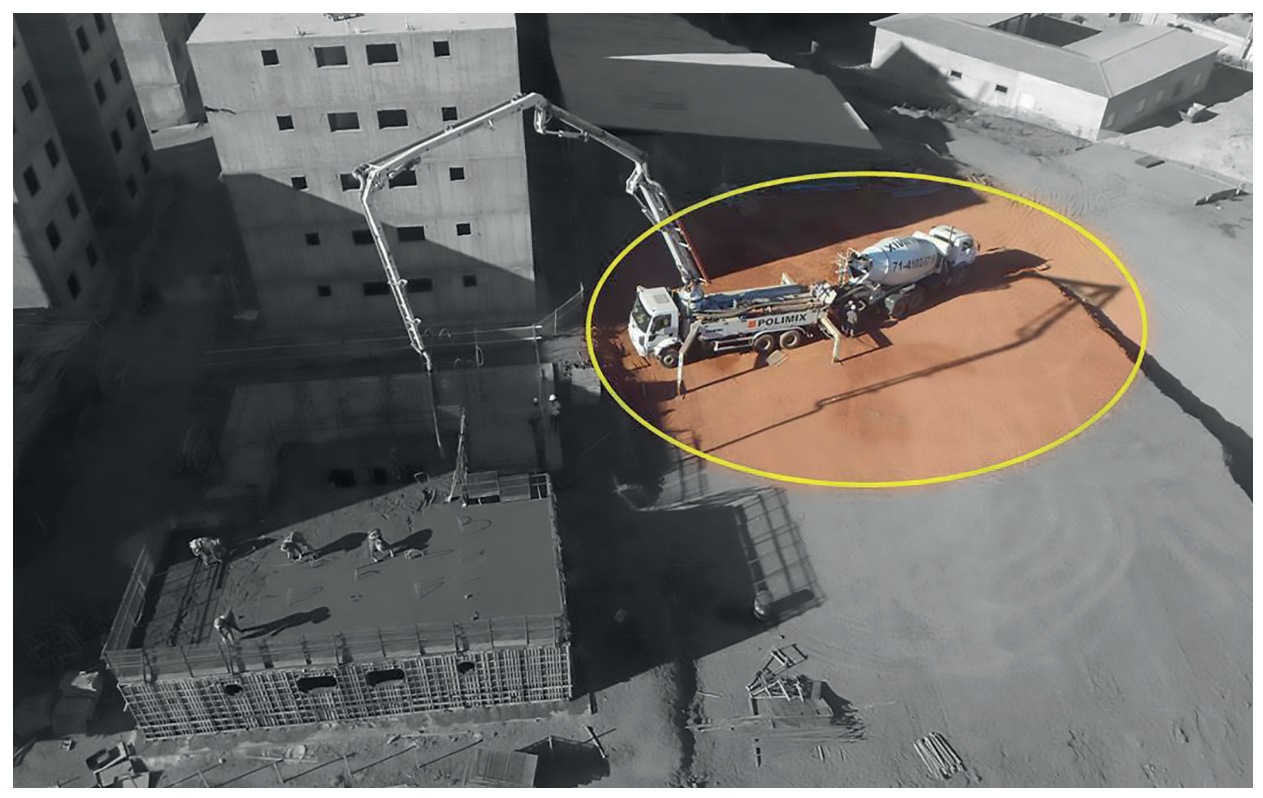

Figura 8 - Ausência de sinalização e isolamento na área de movimentação de carga.

Tabela 5 - Itens avaliados no Checklist de Segurança (Estudo B).

\begin{tabular}{llcccc}
\hline Item & Critério de segurança & 1a Visita & 2a Visita & 3a Visita & 4a Visita \\
\hline 1) Tipo de Captura \# 1. Visualização geral (Overview) & & & \\
\hline 1.5 & $\begin{array}{l}\text { As rotas internas deverão estar limpas e } \\
\text { desobstruídas }\end{array}$ & $\times$ & $\times$ & $\times$ & $\times$ \\
\hline 1.6 & $\begin{array}{l}\text { Os materiais estão armazenados de } \\
\text { forma adequada }\end{array}$ & $\times$ & $\times$ & $\times$ & $\times$ \\
\hline
\end{tabular}

2) Tipo de Captura\# 2.Visualização de Altitudes Intermediárias (Medium Altitude View)

\begin{tabular}{lllll}
\hline 2.1 & $\begin{array}{l}\text { Plataforma de proteção (bandejas) } \\
\text { instalada em toda a periferia isenta de } \\
\text { sobrecarga não prevista e com forração } \\
\text { do piso completa }\end{array}$ & $\times$ & $\times$ & $\times$ \\
\hline 2.6 & $\begin{array}{l}\text { No ponto de descarga da calha para } \\
\text { remoção dos entulhos, existe dispositivo } \\
\text { com fechamento }\end{array}$ & & & \\
\hline Legenda: & $\begin{array}{l}\text { Item } \\
\text { conforme }\end{array}$ & Item \\
não conforme & & - & Não Visualizado
\end{tabular}


Tabela 5 - Continuação.

Item

Critério de segurança

1a Visita 2a Visita 3a Visita 4a Visita

3) Tipo de Captura\# 3. Visualização aproximada por tipo de serviço (Close up view)

3.2) Concretagem e Alvenaria

\begin{tabular}{ll}
\hline 3.2.4 & $\begin{array}{l}\text { As barras ou telas de aço estão } \\
\text { armazenadas em camadas }\end{array}$ \\
\hline 3.2 .8 & $\begin{array}{l}\text { Os trabalhadores expostos aos serviços } \\
\text { de forma, armação, concretagem ou } \\
\text { alvenaria externa estão protegidos contra } \\
\text { queda }\end{array}$
\end{tabular}

3.3) Operação de Equipamentos

\begin{tabular}{lllll}
\hline 3.3.1 & $\begin{array}{l}\text { Sinalização e isolamento da área de } \\
\text { movimentação de carga e descarga }\end{array}$ & $\times$ & $\times$ \\
\hline 3.3 .2 & $\begin{array}{l}\text { Isolamento e delimitação das áreas de } \\
\text { carga e descarga de materiais e raio } \\
\text { de movimentação de gruas, guinchos, } \\
\text { guindastes, etc. }\end{array}$ & $\times$ & $\times$ & $\times$ \\
\hline
\end{tabular}

3.4) Fachada

\begin{tabular}{lllll}
\hline 3.4 .1 & $\begin{array}{l}\text { Os andaimes suspenso ou balancim } \\
\text { devem estar niveladas }\end{array}$ & Os trabalhadores que se encontram na \\
fachada estão usando EPI
\end{tabular}

Na Obra B, foi possível identificar não conformidades quanto à falta de limpeza e organização do canteiro, também se observou a obstrução dos acessos de equipamentos e pedestres (Figura 9), falhas na forração dos pisos das bandejas primárias e secundárias (Figura 10) e acúmulo de materiais e sobrecarga sobre tais locais (Figura 11), além de falhas nos guarda-corpos, comprometendo sua função de proteção contra queda (Figura 12).

Em ambos os estudos, observou-se o uso irregular do EPI, principalmente para as atividades realizadas em locais de menor visibilidade. Tal condição evidencia falhas no processo de inspeção e monitoramento dos atos e condições inseguras por parte da empresa responsável pela execução do serviço, comprovando assim a necessidade de tecnologias que favoreçam a visualização das condições de trabalho. 


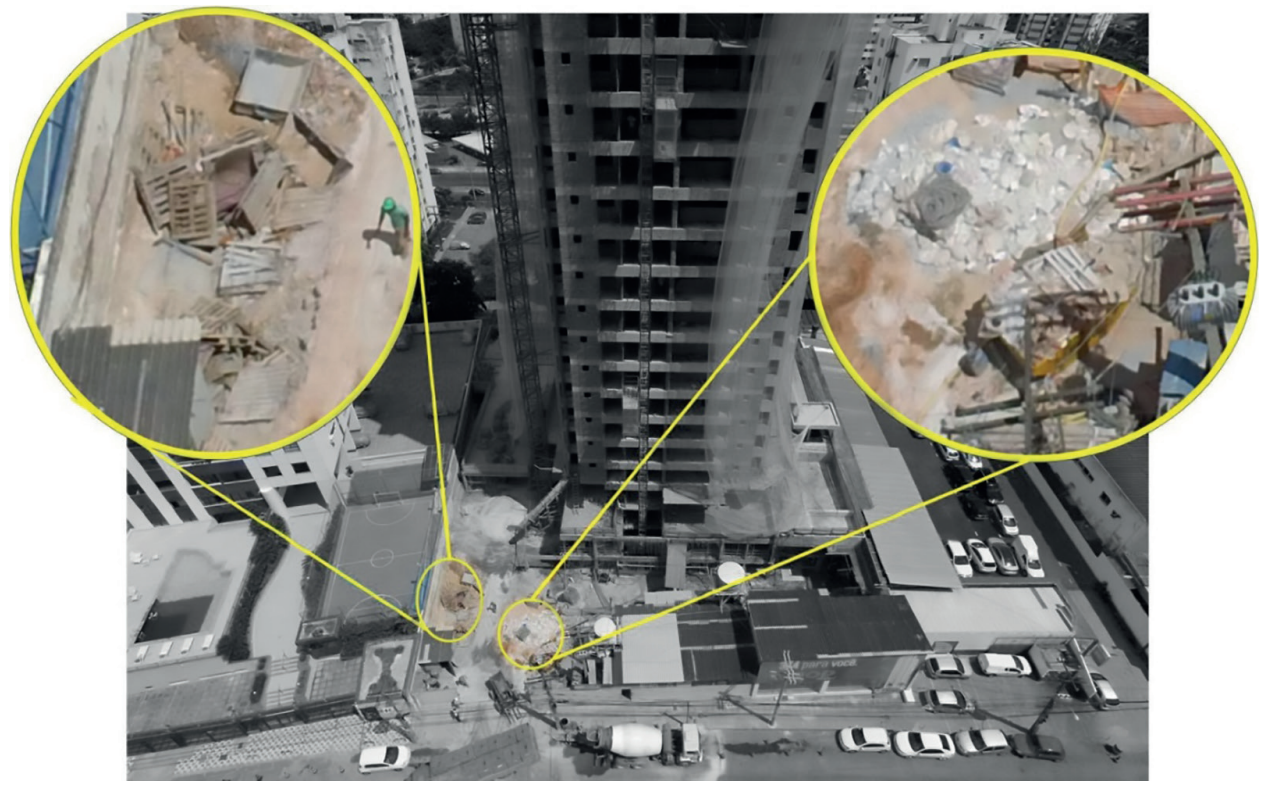

Figura 9 - Acúmulo de resíduos na área de acesso dos trabalhadores.

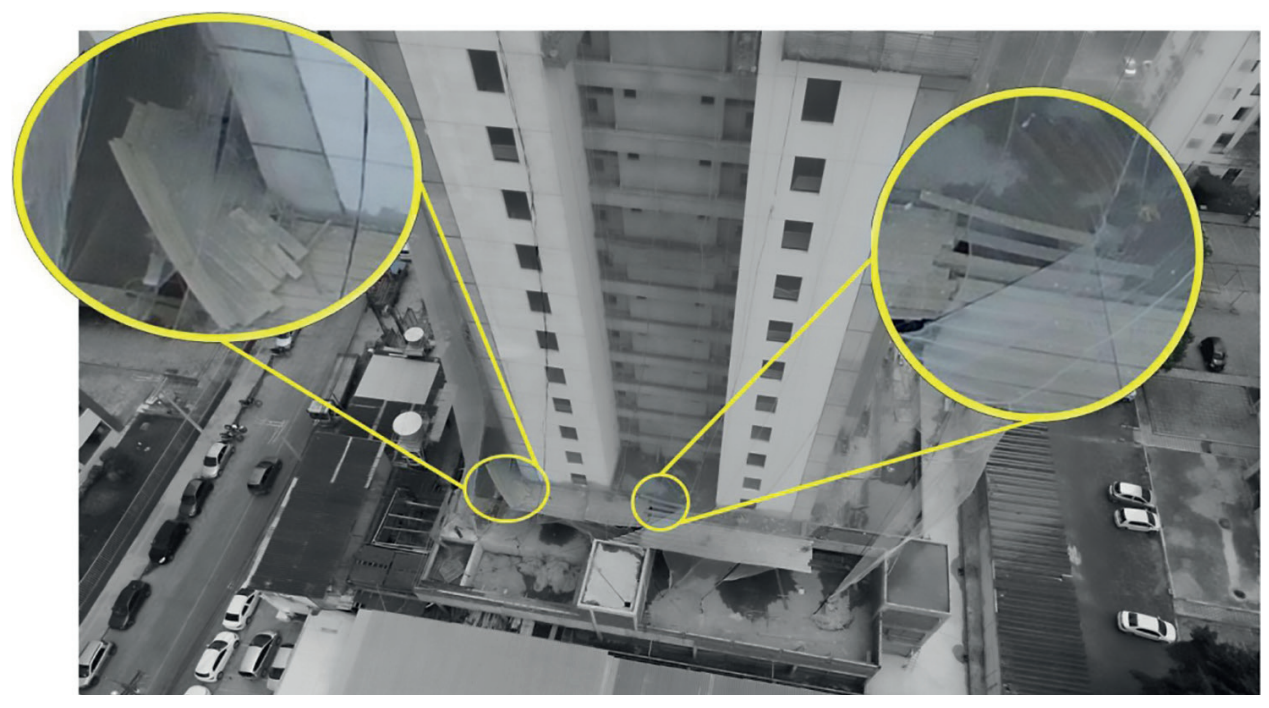

Figura 10 - Plataforma de proteção primária com forração do piso inadequado. 


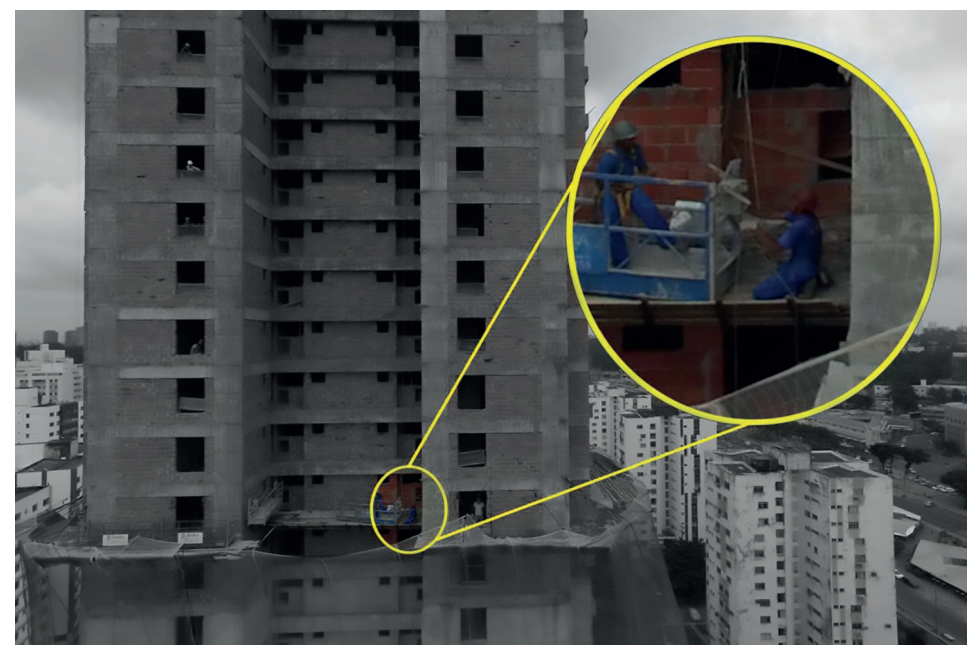

Figura 11 - Plataforma de proteção com sobrecarga não prevista (balancim apoiado sobre a plataforma).

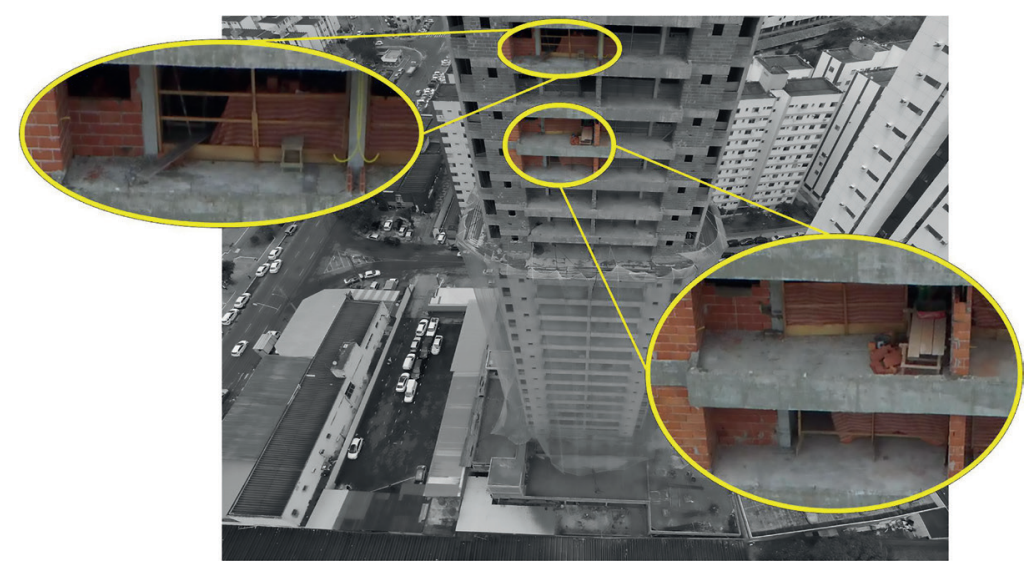

Figura 12 - Guarda corpos com estrutura danificada.

\subsection{Discussões}

O desempenho do equipamento DJl Phantom 3 Advanced satisfez as necessidades para a atividade de inspeção de segurança em termos de autonomia de voo, de estabilidade do VANT, da confiabilidade do sistema e da facilidade de uso. No total, 26 voos foram realizados sem maiores problemas que pudessem causar danos a bens ou pessoas. Além disso, o uso do VANT para inspeção não provocou interferências 
significativas nas atividades desenvolvidas no Canteiro A. Entretanto, no Canteiro B, devido à restrição de espaço para voo, foi solicitada a paralisação da grua, a fim de evitar choques com a aeronave e a ocorrência de acidentes. Em ambos os estudos, antes do início de toda aquisição de dados com o VANT, optou-se pela comunicação via rádio sobre a realização dos voos, para conscientizar os trabalhadores.

No geral, quanto ao potencial de visualização, a Obra A obteve um melhor desempenho (87\% dos itens foram visualizados) comparado à Obra B (60\% dos itens foram visualizados). Tal resultado está diretamente associado a um conjunto de fatores, tais como:

- Condições físicas do canteiro: a Obra A situava-se em região de baixa densidade populacional (permissão de voo com altitude máxima de $120 \mathrm{~m}$ ). Além disso, o Canteiro A possuía área para pouso e decolagem com segurança, enquanto no Canteiro B, os voos eram realizados a partir de áreas externas a ele, mediante autorização de terceiros;

- Barreiras físicas: o canteiro da Obra A quase não dispunha de barreiras físicas, como postes, árvores, fiações elétricas, e outras edificações, já o Canteiro B situava-se em centro urbano, rodeado por outras edificações;

- Fatores meteorológicos: não houve problemas devido a fortes ventos na Obra A, apenas na B;

- Fatores tecnológicos: em ambos os estudos de caso, houve perda do enlace de comando (perda de conexão) entre a estação de controle e a aeronave, no entanto, tal fato não impactou a atividade de inspeção, visto que o dispositivo contra falhas presente na aeronave utilizada (Return to home) funcionou satisfatoriamente;

- Fator humano: para ambos os estudos verificou-se a necessidade de treinamento quanto ao uso da tecnologia, além do conhecimento prévio sobre as questões segurança do canteiro.

Embora se tenha observado uma menor quantidade de itens visualizados na Obra B, ela destaca-se por um maior número de não conformidades identificadas. Além disso, observou-se a necessidade de métodos de inspeção para atividades críticas, como serviços em fachadas, visto a dificuldade de inspeção de tais itens na Obra B por meio de métodos tradicionais.

Referente à análise de não conformidade, foi perceptível o impacto de um sistema de gestão da saúde e segurança, no qual, apesar de diferenças observadas quanto à estruturação de tais sistemas em cada uma das obras, constata-se ainda falhas no processo de inspeção de ambas. A obra A, por possuir sistema de gestão mais consolidado, obteve melhor resultado do que a obra $B$, que não possuía sistema de gestão da segurança definido. 
A Tabela 6 apresenta o resumo dos fatores positivos e das barreiras relativos ao uso da tecnologia VANT para inspeção de segurança em canteiros de obras.

Tabela 6 - Fatores positivos e barreiras quanto ao uso do VANT para inspeção.

\begin{tabular}{|c|c|c|}
\hline Aspectos & Benefícios & Barreiras \\
\hline $\begin{array}{l}\text { Procedimentos e } \\
\text { tecnologia VANT - } \\
\text { Planejamento da } \\
\text { Missão }\end{array}$ & $\begin{array}{l}\text { - Identificação dos pontos de } \\
\text { interesse relativos à segurança. }\end{array}$ & $\begin{array}{l}\text { - Barreiras físicas (edifícios, } \\
\text { postes, árvores, fiações } \\
\text { elétricas, entre outros); } \\
\text { - Condições climáticas (chuva e } \\
\text { ventos fortes). }\end{array}$ \\
\hline $\begin{array}{l}\text { Procedimentos } \\
\text { e tecnologia } \\
\text { VANT - Coleta e } \\
\text { processamento de } \\
\text { dados }\end{array}$ & $\begin{array}{l}\text { - Redução do tempo de inspeção } \\
\text { devido à flexibilidade no } \\
\text { monitoramento mútuo de } \\
\text { diferentes atividades; } \\
\text { - Simplificação das etapas } \\
\text { de inspeção por meio da } \\
\text { eliminação do excesso de coleta } \\
\text { manual (redução de pessoal); } \\
\text { - Tecnologia de fácil uso; } \\
\text { - Redução da variabilidade com a } \\
\text { padronização dos dados. }\end{array}$ & $\begin{array}{l}\text { - Requisitos das regulamentações } \\
\text { (ex. limite de altitude); } \\
\text { - Treinamento de operação do } \\
\text { VANT para piloto e observador; } \\
\text { - Conhecimento prévio de } \\
\text { segurança para o piloto e } \\
\text { observador; } \\
\text { - Grande base de dados de ativos } \\
\text { visuais; } \\
\text { - Visualização de áreas internas } \\
\text { limitada. }\end{array}$ \\
\hline $\begin{array}{l}\text { Procedimentos e } \\
\text { tecnologia VANT - } \\
\text { Análise de dados } \\
\text { e proposição de } \\
\text { melhorias }\end{array}$ & $\begin{array}{l}\text { - Aumento da transparência das } \\
\text { condições inseguras; } \\
\text { - Informações detalhadas sobre } \\
\text { condições inseguras e seguras; } \\
\text { - Registro das não conformidades } \\
\text { de segurança e das boas } \\
\text { práticas, permitindo a análise } \\
\text { de diferentes perspectivas; } \\
\text { - Utilização de indicadores e } \\
\text { informações para tomada de } \\
\text { decisão; } \\
\text { - Utilização dos ativos visuais e } \\
\text { dos resultados de inspeção para } \\
\text { a educação de segurança. }\end{array}$ & $\begin{array}{l}\text { - Análise manual dos ativos } \\
\text { visuais; } \\
\text { - Necessidade de melhorar } \\
\text { o tempo de resposta aos } \\
\text { trabalhadores, visando } \\
\text { feedback em tempo real } \\
\text { (interação direta entre VANT e } \\
\text { trabalhadores); } \\
\text { - Necessidade de fornecer } \\
\text { informações em tempo real } \\
\text { para segurança (interação } \\
\text { direta entre o VANT e equipe de } \\
\text { segurança). }\end{array}$ \\
\hline $\begin{array}{l}\text { Sistema de } \\
\text { Segurança e Pessoas }\end{array}$ & $\begin{array}{l}\text { - Viabilidade de custo-benefício } \\
\text { do VANT (tecnologia de } \\
\text { baixo custo de aquisição e } \\
\text { manutenção); } \\
\text { - Potencial de melhoria do } \\
\text { comportamento do trabalhador } \\
\text { em relação à segurança. }\end{array}$ & $\begin{array}{l}\text { - Integração do VANT ao sistema } \\
\text { de gestão de saúde e segurança } \\
\text { da obra; } \\
\text { - Resistência à adoção de novas } \\
\text { tecnologias pela construção } \\
\text { civil; } \\
\text { - Sistema mal estruturado } \\
\text { de gestão da segurança em } \\
\text { canteiro de obras. }\end{array}$ \\
\hline
\end{tabular}


Dentre as principais recomendações para o uso do VANT para inspeção de segurança em canteiros, podem ser destacadas:

\section{- Planejamento:}

- Analisar os critérios de segurança para voo, estabelecidos pelas agências reguladoras de aviação no país;

- Conhecer os elementos principais sob a perspectiva de segurança do canteiro e os riscos associados a cada serviço;

- Realizar campanha com os trabalhadores em relação ao uso de VANT para inspeção de segurança;

- Definir os processos críticos possíveis de serem monitorados com o VANT sob a perspectiva de segurança;

- Analisar previamente as possíveis interferências físicas aos voos, tais como gruas, árvores, postes, entre outros;

- Definir a sequência dos pontos a serem monitorados;

- Atribuir responsabilidades quanto às etapas do protocolo de inspeção;

- Garantir treinamento do piloto e observador quanto ao uso do VANT e quanto aos itens e critérios de segurança que serão inspecionados;

- Definir indicadores para avaliar a eficácia do processo de inspeção.

\section{- Coleta e processamento de dados:}

- Padronizar o processo de coleta de dados (Checklist de Missão e Checklist de Segurança) com o objetivo de simplificar o processo, eliminar a coleta de informações redundantes e reduzir o tempo de inspeção;

- Estabelecer a periodicidade da inspeção conforme a necessidade de monitoramento dos processos, pois a inspeção com VANT tem caráter pontual, ou seja, registra as irregularidades para um determinado momento;

- Realizar o processamento dos dados logo após os voos, para possibilitar a análise imediata.

- Análise de dados e proposição de melhorias:

- Promover a análise aprofundada a partir dos ativos visuais coletados, visto que os mesmos possibilitam a reanálise por diferentes perspectivas, viabilizando a proposição de medidas preventivas;

- Promover encontros periódicos com os colaboradores e equipe gerencial para apresentação e discussão dos resultados obtidos, visando aumentar a eficácia no atendimento das metas estabelecidas; 
- Desenvolver clima e cultura de segurança com ênfase na observação das não conformidades, baseado em registros visuais, a fim de contribuir com a mudança de postura dos colaboradores;

- Usar os ativos visuais para treinamento dos colaboradores relativos a atos e condições inseguras, baseado em exemplos da realidade vivenciada pelos próprios trabalhadores.

\section{Conclusões}

Este estudo teve por objetivo avaliar a aplicabilidade da tecnologia VANT para inspeção de segurança em canteiros de obra, focando na capacidade de identificação de não conformidades que podem fornecer condições inseguras ao trabalhador. Os resultados encontrados apontam que o VANT permite uma melhor visualização das condições de trabalho, principalmente em locais com limitações de acesso (fachadas, coberturas e telhados).

Umas das contribuições deste estudo é o desenvolvimento de um conjunto de procedimentos para inspeção de segurança em canteiros de obra com VANT (protocolo de inspeção apresentado na Figura 2). Para tal, foram adaptados formulários desenvolvidos por Irizarry, Costa e Kim (2015), tais como: Formulário de Planejamento e Checklist de Missão. O Checklist de Segurança por tipo de captura foi adaptado às normas de segurança brasileiras (NR 18 - Condições e meio ambiente de trabalho na indústria da construção e NR 35 - Trabalho em altura). Para a análise dos dados, foi desenvolvida uma base de dados, além de métricas relacionadas com a visualização e não conformidade dos itens de segurança.

Além disso, os tipos de captura apresentam uma maneira inovadora para inspecionar itens de segurança, fornecendo informações em diferentes perspectivas. O Overview fornece informações gerais sobre a organização e limpeza do canteiro, o Medium View e o Close Up fornecem informações mais específicas sobre os itens de segurança relacionados a equipamentos de proteção coletiva e individual, além de possibilitar a inspeção de atividades específicas, como serviços em telhado.

Ainda há uma lacuna sobre como integrar de maneira efetiva a tecnologia VANT no processo de inspeção de segurança dos canteiros. Há uma expectativa de que o VANT possa impactar na melhor utilização dos recursos disponíveis para as inspeções. No entanto, novos estudos são necessários para avaliar o impacto do VANT no processo de inspeção de segurança de forma sistemática, com foco no feedback rápido, permitindo ações corretivas imediatas, reduzindo o tempo e simplificando o processo de inspeção de segurança. 


\section{Referências}

AGÊNCIA NACIONAL DE AVIAÇÃO CIVIL (ANAC). Regulamento Brasileiro da aviação Civil Especial (RBAC-E no94) .Brasilia, DF: 2015. Disponível em: <http://www2.anac.gov.br/ transparencia/audiencia/2015/aud13/anexol.pdf>. Acesso em: set. 2015.

BRASIL. Ministério do Trabalho e Emprego. Norma Regulamentadora 18 (NR-18): Condições e Meio Ambiente de Trabalho na Indústria da Construção. Rio de Janeiro: 2015. Disponível em: <http://trabalho.gov.br/seguranca-e-saude-no-trabalho/normatizacao/ normas-regulamentadoras/norma-regulamentadora-n-18-condicoes-e-meio-ambientede-trabalho-na-industria-da-construcao>. Acesso em: nov. 2015.

BRASIL. Ministério do Trabalho e Emprego. Norma Regulamentadora 35 (NR-35): Trabalho em Altura. Rio de Janeiro: 2014. Disponível em: < http://portal.mte.gov.br/images/ Documentos/SST/NR/NR4.pdf>. Acesso em: nov. 2015.

CAMBRAIA, F.B.; SAURIN, T.; FORMOSO, C. T. Identification, analysis and dissemination of information on near misses: a case study in the construction industry. Safety Science, v.48, p.91-99, 2010.

EMELIANOV, S.; BULGAKOW, A.; SAYFEDDINE, D. Aerial laser inspection of buildings facades using quadrator. Precedia Engineering, v. 85, p. 140-146, 2014.

ESCHMANN, C.; KUO, C.M.; KUO, C.H.; BOLLER, C. Unmanned aircraft systems for remote building inspection and monitoring. In: 6th European workshop on structural health monitoring. 2012. Proceedings...

IRIZARRY, J.; COSTA, D.B. Exploratory Study of potential Applications of Unmanned Aerial Systems for Construction Management Tasks. Journal of Management in Engineering, v. 32, n. 3, 2016.

IRIZARRY, J.; COSTA, D.B.; KIM, S. Evaluation of Unmanned Aerial Systems in Construction Safety Applications: A Case Study at Unilever Manufacturing Facility in Independence, MO. Final Report. Atlanta: Georgia Institute of Technology, School of Building Construction.2015.

IRIZARRY, J.; GHEISARI, M.; WALKER, B. N. Usability assessment of drone technology as safety inspection tools. Journal of Information Technology in Construction (ITcon), 2012, 17, 194-212. Proceedings...

KIM, S.; IRIZARRY, J. Exploratory study on factors influencing UAS performance on highway construction projects: as the case of safety monitoring systems. In: Conference on Autonomous and Robotic Construction of Infrastructure, Ames, 2015. Proceedings...

KIM, Y.S.; OH, S.W.; CHO, Y.K.; SEO, J.W. A PDA and Wireless Web-Integrated System for Quality Inspection and Defect Management of Apartment Housing Projects. Automation in Construction, v. 17, n. 2, p. 163-179. 2008. DOI: 10.1016/j.autcon.2007.03.006

LIN, K.-Y.; TSAI, M.-H.; GATTI, U.C.; LIN, J.J.-C.; LEE, C.-H.; KANG, S.-C. A User-Centered Information and Communication Technology (ICT) Tool to Improve Safety Inspections. Automation in Construction, v. 48, n. 1, p. 53-63, 2014. DOI: 10.1016/j.autcon.2014.08.012 
METNI, N.; HAMEL, T. A UAV for bridge inspection: visual servoing control law with orientation limits. Automation in Construction, v. 17, n. 1, p. 3-10, 2007.

MORGENTHAL, G.; HALLERMANN, N. Quality Assessment of Unmanned Aerial Vehicle (UAV) Based Visual Inspection of Structures. Advances in Structural Engineering, v. 17, n. 3, p. 289-302, 2014. Proceedings...

PARK, C.S.; LEE, O,-S.;WANG, W. A framework for proactive construction defect management using BIM, augmented reality and ontology-based data collection template. Automation in Construction, v. 33, n. 1, p. 61-71. 2013. DOI: 10.1016/j.autcon.2012.09.010

SHRESTHA, P.; YFANTIS, E.; SHRESTHA, K. Construction safety visualization. University of Nevada, Las Vegas (UNLV), 89154, U.S.A. 2011.

THEMISTOCLEOUS, K.; NEOCLEOUS K.; PILAKOUTAS, K.; HADJIMITSIS, D.G. Damage assessment using advanced non-intrusive inspection methods: Integration of Space, UAV, GPR and Field Spectroscopy. Proc. 2th International Conference on Remote Sensing and Geoinformation of the Environment. V. 9229. Paphos, Cyprus, 2014. Proceedings...

WOODCOCK, K. Model of safety inspection. Safety Science. V. 62, pp. 145-156, 2014.

ZHANG, C. Development of a UAV-based remote sensing system for unpaved road condition assessment. In: American Society for Photogrammetry and Remote Sensing Annual Conference, Portland, Oregon, 2008. Proceedings... 